\title{
Central Adaptation to Odorants Depends on PI3K Levels in Local Interneurons of the Antennal Lobe
}

\author{
Angel Acebes, ${ }^{1}$ Jean-Marc Devaud, ${ }^{2}$ Mercedes Arnés, ${ }^{1}$ and Alberto Ferrús ${ }^{1}$ \\ ${ }^{1}$ Cajal Institute, Consejo Superior de Investigaciones Científicas, Madrid 28002, Spain, and ${ }^{2}$ Centre de Recherches sur la Cognition Animale, CNRS UMR \\ 5169, Université Paul Sabatier, Toulouse 31062, France
}

We have previously shown that driving PI3K levels up or down leads to increases or reductions in the number of synapses, respectively. Using these tools to assay their behavioral effects in Drosophila melanogaster, we showed that a loss of synapses in two sets of local interneurons, GH298 and krasavietz, leads to olfaction changes toward attraction or repulsion, while the simultaneous manipulation of both sets of neurons restored normal olfactory indexes. We show here that olfactory central adaptation also requires the equilibrated changes in both sets of local interneurons. The same genetic manipulations directed to projection (GH146) or mushroom body (201Y, MB247) neurons did not affect adaptation. Also, we show that the equilibrium is a requirement for the glomerulus-specific size changes which are a morphological signature of central adaptation. Since the two sets of local neurons are mostly, although not exclusively, inhibitory (GH298) and excitatory (krasavietz), we interpret that the normal phenomena of sensory perception, measured as an olfactory index, and central adaptation rely on an inhibition/excitation ratio.

\section{Introduction}

Learning and memory are brain properties based on changes in synaptic activity within specific circuits and time sequences as a result of experience. These features of the classical definition have not incorporated yet the role of changes in the number of synapses mainly because quantitative synaptic studies are scant and difficult.

The Drosophila olfactory system is suitable to study simple forms of learning and the synaptic changes that sustain them (Busto et al., 2010). In the antennal lobe (AL) glomeruli, olfactory sensory neurons (OSNs) contact projection neurons (PNs) and local interneurons (LNs). Two classes of LNs interconnect glomeruli, GABAergic interneurons (iLNs), which provide inhibition to OSNs and PNs (Wilson et al., 2004) and cholinergic interneurons (eLNs), which allow lateral excitation between glomeruli and contact also to PNs (Olsen et al., 2007; Shang et al., 2007; Yaksi and Wilson, 2010). Once processed by the AL, olfactory information is conveyed by PNs to the mushroom bodies (MBs) and lateral horn (Root et al., 2007; Tanaka et al., 2009). Although this connectivity is considered structurally stable (Berdnik et al., 2006), activity leads to long-lasting morphological changes. Flies exposed to high concentration of odorants during 4-5 d develop odorant-specific reduced avoidance that does not alter OSN responses, it persists for days, it does not appear to be reversible, it includes glomerulus-specific changes in volume and it is depen-

Received June 10, 2011; revised Nov. 3, 2011; accepted Nov. 7, 2011.

Research was funded by grants from the Ministry of Research (BFU2009-12410/BMC), a Network of Excellence award from the Comunidad de Madrid (OLFACTOSENSE) (SEM-0255-2006-02), Fundación Reina Sofía, and Fundación Areces.

Correspondence should be addressed to Angel Acebes, Cajal Institute, C.S.I.C., Ave. Dr. Arce 37, 28002 Madrid, Spain. E-mail: a.acebes@cajal.csic.es.

DOI:10.1523/JNEUROSCI.2921-11.2012

Copyright $\odot 2012$ the authors $\quad 0270-6474 / 12 / 320417-06 \$ 15.00 / 0$ dent on cAMP signaling. We have defined this effect as "central adaptation" (Devaud et al., 2001).

To better understand the neural bases of this form of experience-dependent plasticity, we took a genetic approach to modify synapse numbers in specific neuron populations. Indeed, we identified in Drosophila a signaling pathway that regulates the number of synapses without affecting the number of neurons (Martín-Peña et al., 2006). Overexpressing phosphoinositide-3kinase (PI3K), or its down-stream element AKT/PKB, leads to an increase in the number of synapses. In contrast, the overexpression of PI $3 \mathrm{~K}^{\mathrm{DN}}$, a dominant-negative form of PI3K, or the glycogen synthase kinase-3 (GSK3), cause synapse number reduction. This pathway is conserved in vertebrate hippocampal neurons (Cuesto et al., 2011). We have previously documented by electron microscopy that these constructs actually change the number of synapses in the targeted local interneurons (Acebes et al., 2011). Here, we addressed two cellular aspects of olfactory central adaptation: (1) which neurons build it? and (2) how does synapse activity relate to this phenomenon? The experimental strategy consisted in driving PI3K constructs in excitatory and/or inhibitory local neurons and measuring the olfactory index as a behavioral read-out under conditions that elicit central adaptation.

\section{Materials and Methods}

Fly strains, behavioral assay and odorants. All strains used here are described previously (Stocker et al., 1997; Acebes et al., 2011). Male flies were exposed during the first $5 \mathrm{~d}$ of adulthood to an odorant $\left(10^{-1} \mathrm{v} / \mathrm{v}\right)$. Controls were exposed to the solvent (paraffin oil). On day 6, a choice assay was performed using a T-maze as described previously (Acebes and Ferrús, 2001). Briefly, the animals could choose during $30 \mathrm{~s}$ between a compartment containing the odorant $\left(10^{-2} \mathrm{v} / \mathrm{v}\right)$ and another containing the solvent (paraffin oil). The olfactory response index (OI) shows the number of flies trapped in the odor-compartment minus the number trapped in the solvent-compartment, divided by the total. Each data 



Figure 1. Morphological effects of overexpressing PI3K constructs. $\boldsymbol{A}-\mathbf{I}$, Confocal images of whole-mount mushroom bodies from anterior $(\boldsymbol{A}-\boldsymbol{C})$ and posterior $(\boldsymbol{D}-\boldsymbol{I})$ views. Neurons were visualized by immunolabeling of the membrane CD8-GFP reporter. In the 201Y-Gal4 domain, UAS-PI3K and UAS-GSK3 caused an increase or decrease, respectively, in the size of the mushroom body lobes $(\boldsymbol{B}, \boldsymbol{C})$ and calyces $(\boldsymbol{E}, \boldsymbol{F})$ compared with control $(\boldsymbol{A}-\boldsymbol{D})$. Equivalent effects were observed in the calyx when PI3K constructs were overexpressed in the MB247-Gal4 domain $(\boldsymbol{G}-\boldsymbol{I})$. $\boldsymbol{J}, \boldsymbol{K}$, Quantification of the effects. $\boldsymbol{L}-\mathbf{Q}$, Morphological effects in PNs of GH146-Gal4 domain. These cells (arrowhead in $\boldsymbol{L}$ ) have their dendrites in several AL glomeruli (outlined in $\boldsymbol{L}$ ) and send their axons to $\mathrm{MB}$ and lateral horn $(\mathrm{LH})$ brain centers (outlined in $\mathbf{0})$. Note the increase $(P I 3 K)$ and reduction $\left(P I 3 K^{D N}\right.$ ) in the size and GFP signal intensity in all targeted areas. Scale bars (in $\left.\boldsymbol{F}\right) \boldsymbol{A}-\mathbf{I}, 75 \mu \mathrm{m}$; (in Q) $L-Q, 50 \mu \mathrm{m}$.

point represents $300-400$ individuals distributed in $15-16$ replicates in random order. Flies that did not make any choice were excluded, and tests where more than one third of the flies did not make any choice were discarded. The odorants used, ethyl butyrate (EB, Fluka Chemicals) and isoamyl acetate (IAA, Sigma), were of the highest purity (>95\%). All experiments were conducted in the dark at $25^{\circ} \mathrm{C}$.
Confocal microscopy and glomerular volume measurements. Male flies subjected to the behavioral assay had their brains dissected in phosphate buffer and mounted unfixed in Vectashield (Vector Labs). Confocal images were acquired on a Leica TCS-SP5 microscope. Serial optical sections $(512 \times 512$ pixels $)$ were taken at $1 \mu \mathrm{m}$ intervals using a $40 \times$ oil-immersion objective and saved as $3 \mathrm{D}$ stacks covering the entire $\mathrm{AL}$. 
$\square$ Control (Paraffin oil) $\square$ Exposed (EB 10 $\left.10^{-1}\right)$ $\square$ Control (Paraffin oil) $\square$ Exposed (IAA 10-1)
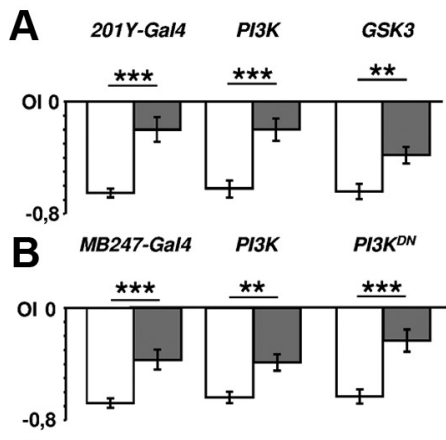

C GH146-Gal4 PI3K PI3K ${ }^{D N}$

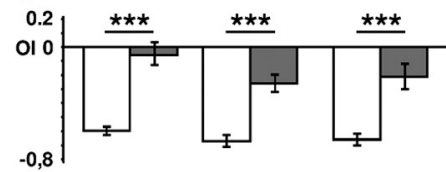

D GH298-Gal4 PI3K GSK3

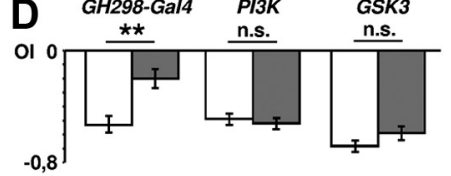

G
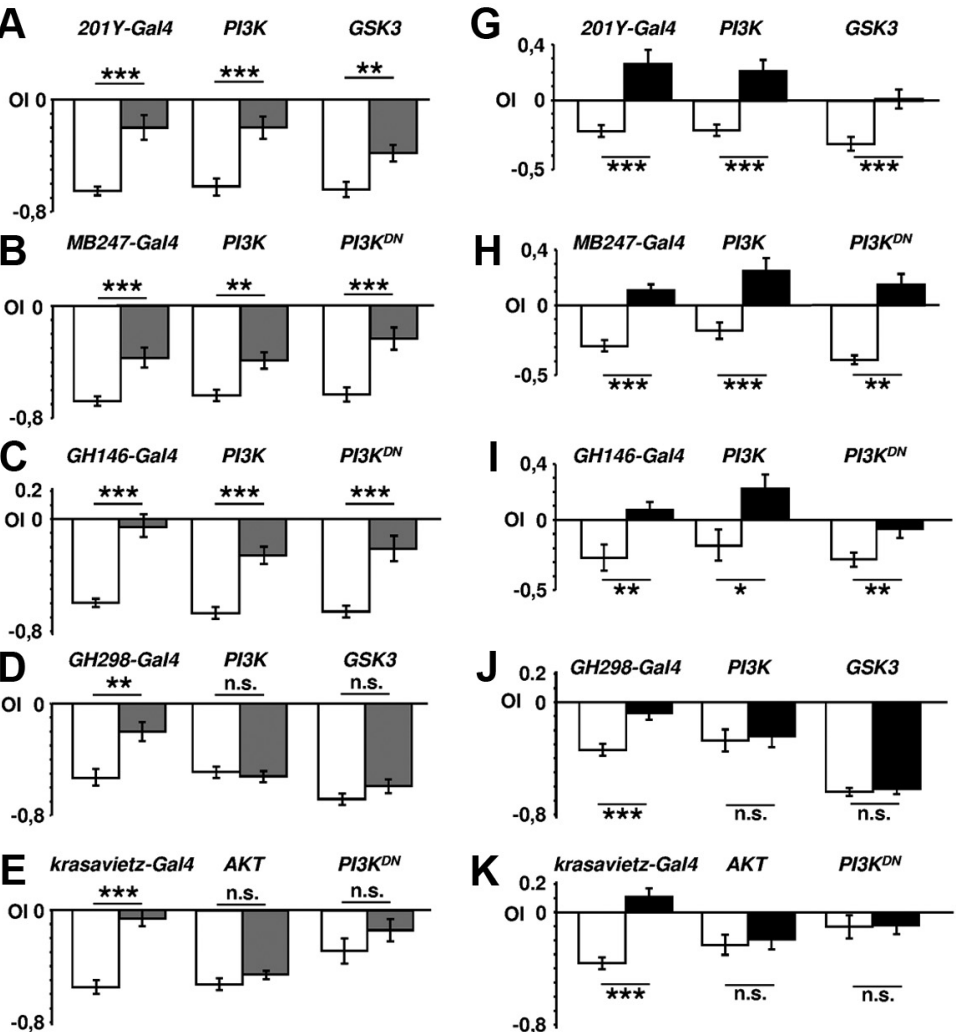

$J_{\text {OI }} 0.2$

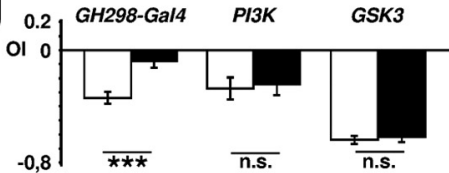

$-0,8 \overline{\star \star \star} \quad \overline{n . s .}$

n.s.

$\mathbf{K}$
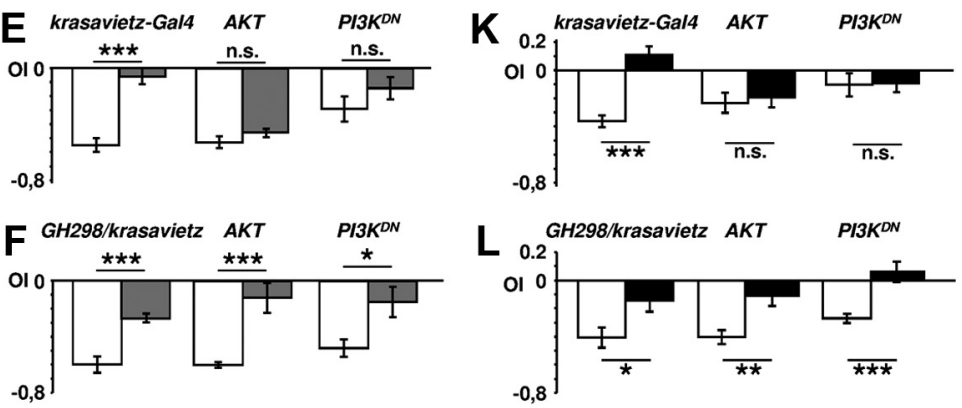

EB 10-2

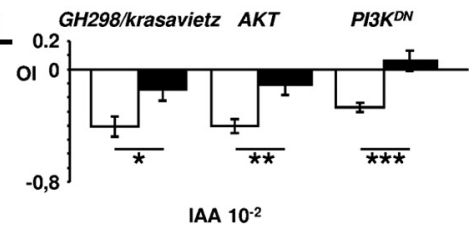

Figure 2. Central adaptation after modification of MB neurons and PNs. $A-L$, Olfactory response index (OI) to $10^{-2} E B(A-F)$ and $10^{-2}$ IAA (G-L) in 6-d-old adults previously exposed to the same odorant $\left(10^{-1}\right)$ (gray and black columns to EB and IAA, respectively) or to paraffin oil (white columns). In all genotypes, preexposed flies were significantly less repelled by the odorant than control individuals. Genotypes were tested randomly and each group of flies was subjected to one odorant concentration choice only. Neither modification in mushroom body neurons (201Y-Gal4 and MB247-Ga/4) $(\boldsymbol{A}, \boldsymbol{H})$ nor modification in projection neurons (GH146) $(C, I)$ affected central adaptation to EB or IAA. However, the effect was prevented when synapse number was putatively increased (PI3K, AKT) or reduced (PI3K $\left.{ }^{\mathrm{DN}}, G S K 3\right)$ in the $G H 298(\boldsymbol{D}, \boldsymbol{J})$ or krasavietz $(\boldsymbol{E}, \boldsymbol{K})$ domains. By contrast, central adaptation was normal when both neuronal domains were modified simultaneously $(\boldsymbol{F}, \boldsymbol{L})$. n.S., Not significant.

Stacks were imported into Amira 3.1 (Mercury Computer Systems) for image analysis. The DM2 and V glomeruli were identified on the published atlas (Laissue et al., 1999). All drawings and calculations were run blind. Laser settings were maintained constant throughout all the genotypes analyzed. Histology and immunostaining procedures were as previously described (Acebes et al., 2011).

Statistical analyses. Data are given in the text as mean \pm SEM, and presented as such in figures, with the exception of Figure 4 (see below), in which dot plots are provided. In most cases, data were analyzed using the Student's two-tailed $t$ test. The Mann-Whitney $U$ test was applied to measurements of glomerular volume. Significance levels are noted as ${ }^{*} p<0.05,{ }^{* *} p<0.001,{ }^{* *} p<0.0001$ in the figures. SPSS and GraphPad Instat 3 software was used throughout.

\section{Results}

PI3K constructs expressed in MB neurons or AL projection neurons do not alter central adaptation

We analyzed the contribution of MB neurons to olfactory adaptation by genetically driving $P I 3 K, G S K 3$ or $P I 3 K^{D N}$ in selected MB neuronal populations. Two Gal4 drivers were used: 201Y, which expresses in $~ 50 \%$, and $M B 247$ which expresses in 25-75\% of MB neurons according to the available reports (Schwaerzel et al., 2002; Aso et al., 2009). The morphological effects due to the expression of the genetic constructs were analyzed using the UAS-CD8-GFP or UAS-n-Synaptobrevin-GFP reporters. Neurons in $201 Y$ and MB247 domains, exhibited increased branching and synaptic reporter expression when $P I 3 K$ was upregulated, while these parameters were reduced when overexpressing $P I 3 K^{D N}$ or GSK3 (Fig. $1 A-K$ ). These effects are consistent with the direct synapse quantification by electron microscopy in the antennal lobe LNs (Acebes et al., 2011) and the ellipsoidal body (Martín-Peña et al., 2006).

To elicit central adaptation, flies were exposed to EB $\left(10^{-1} \mathrm{v} / \mathrm{v}\right)$ (Fig. $\left.2 A, B\right)$ or IAA (Fig. 2G,H) and tested for their response to the same odorant $\left(10^{-2} \mathrm{v} / \mathrm{v}\right)$. Control flies exposed to paraffin oil displayed avoidance (Fig. $2 A, B, G, H$, white bars). By contrast, odorant-exposed flies showed reduced avoidance (gray bars in Fig. $2 A, B$, black bars in $2 G, H)$. Adaptation of the responses was also observed when either $P I 3 K$ or $P I 3 K^{D N} / G S K 3$ were overexpressed in these $\mathrm{MB}$ domains. Thus, we conclude that the synaptic changes elicited in the MBs do not have detectable consequences on adaptation.

To analyze the PNs, we used the GH146-Gal4 driver (Stocker et al., 1997). This line labels $\sim 90$ neurons that express choline acetyltransferase and receive synaptic input from OSNs, eLNs and iLNs (Yaksi and Wilson, 2010). As above, the expression of PI3K and GSK3 constructs elicited the expected morphological effects in GH146 neurons (Fig. $1 L-Q$ ). However, similarly to MB neurons, PI3K/ GSK3 in PNs did not affect adaptation to EB (Fig. 2C) or IAA (Fig. 2I).

\section{Synaptogenic changes in AL local neurons prevent central adaptation}

The LNs synapse throughout the AL glomeruli where they receive input from OSNs. They mediate inhibition (iLNs) or excitation (eLNs), depending on which neurotransmitter they express: GABA (Stocker et al., 1997; Olsen et al., 2010) or acetylcholine (Ach) (Shang et al., 2007; Huang et al., 2010), respectively. Recently, electrical synapses have been revealed in the AL providing functional coupling between LNs and PNs as well as among LNs (Yaksi and Wilson, 2010). To address the role of LNs in central adaptation, we used two drivers: GH298-Gal4 expressed in 30, mostly inhibitory, LNs (Stocker et al., 1997); and krasavietz-Gal4, expressed in a small subset of $\sim 8$, mostly excitatory, LNs. Our krasavietz line is similar to the one originally described (Shang et al., 2007) while it differs from another line with the same name, 
A
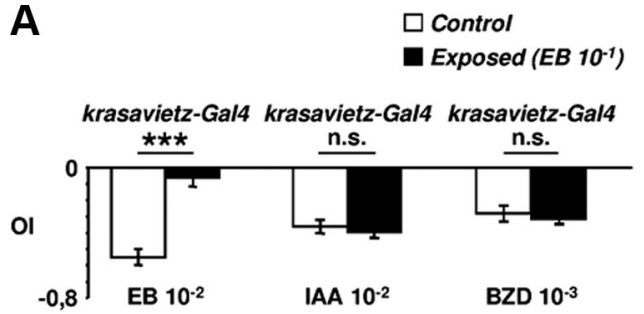

B
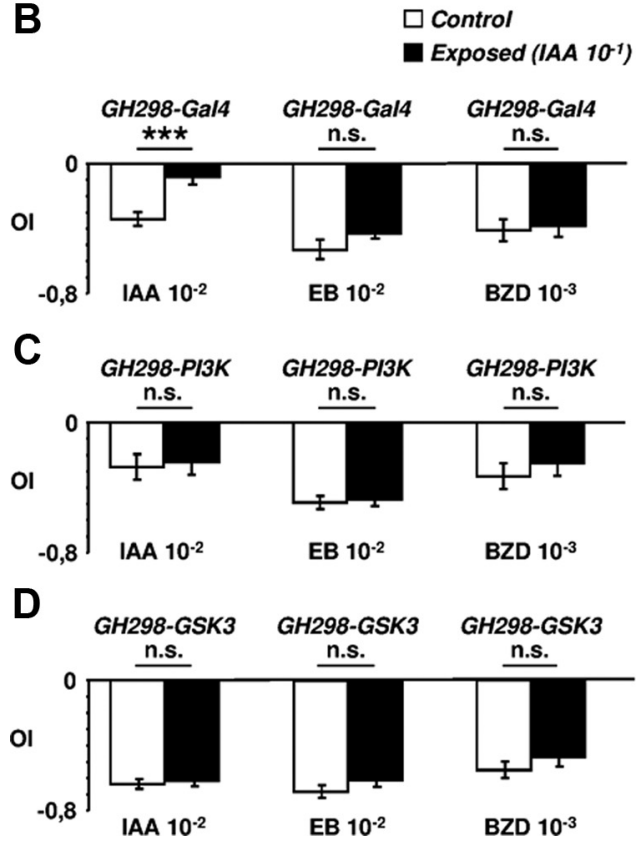

Figure 3. Olfactory central adaptation is odor-specific. $A$, The krasavietz-Gal4 flies were exposed to $10^{-1} \mathrm{~EB}$ (black bars) or to paraffin oil (white bars) and tested with $10^{-1}$ IAA or $10^{-3}$ benzaldehyde (BZD). $\boldsymbol{B}$, The same test using GH298-Gal4 flies exposed to IAA. Note that in both cases the phenomenon develops for the odorant of exposure only. C, D, The increase (PI3K) or reduction (GSK3) of synapses in the GH-298 neurons (iLNs) prevents the establishment of central adaptation following the exposure to IAA and the effect is general to the three odorants tested. n.s., Not significant.

recently reported (Chou et al., 2010; Seki et al., 2010), that we renamed Line 9 (Acebes et al., 2011).

Overexpression of $P I 3 K, A K T, G S K 3$ or $P I 3 K^{D N}$ under the control of GH298 or krasavietz did affect central adaptation (Fig. $2 D, K)$. Flies expressing any of these constructs avoided the odorants as strongly as controls which had been exposed only to the solvent. That is, central adaptation to EB (Fig. $2 D, E$ ) or IAA (Fig. $2 J, K$ ) was prevented in flies with modified LNs, either excitatory or inhibitory. This effect was independent of the driven construct, PI3K/AKT or $P I 3 K^{D N} / G S K 3$. In addition, we performed cross-adaptation assays (Fig. $3 A, B$ ). Flies were exposed to either $\mathrm{EB}$ or IAA and tested for their avoidance from IAA or EB, respectively. We also included another aversive stimulus, $10^{-3}$ benzaldehyde, in the tests. Control flies developed the phenomenon only to the experienced odorant, as previously shown (Devaud et al., 2001), indicating that cross-adaptation did not occur, at least among the odorants tested. In the PI3K/AKT/GSK3-expressing genotypes, in which central adaptation did not develop, crossadaptation was also absent, as expected (Fig. $3 C, D$ ). Thus, we conclude that the reduction of either excitatory or inhibitory synapses in AL local interneurons prevents central adaptation and that LNs are the key cells for this phenomenon.
The ratio of LN synapses controls central adaptation

Recently, we reported that sensory perception was modified in opposite directions - shift toward attraction or repulsion- depending on which LNs had been targeted (Acebes et al., 2011). Also, we reported that normal olfactory indexes could be restored if the reduction of synapses was achieved simultaneously in eLNs and iLNs (Acebes et al., 2011). Thus, we asked whether a balance between the two sets of neurons might also be crucial also for central adaptation. To this end, we combined GH298 and krasavietz drivers to elicit the expected increase $(A K T)$ or reduction $\left(P I 3 K^{D N}\right)$ of the number of synapses in both sets of neurons simultaneously. In these flies, adaptation to EB (Fig. $2 F$ ) or IAA (Fig. $2 L$ ) was established normally. These results strongly suggest that olfactory central adaptation depends on the eLN/iLN activity ratio rather than on the absolute number of either type of synapses.

\section{Central adaptation involves glomerulus-specific} morphological plasticity

As central adaptation correlates with glomerulus-specific size changes (Devaud et al., 2001; Sachse et al., 2007), we reasoned that the behavioral phenotypes should include coherent morphological changes in glomerular volume. We measured two identified glomeruli, DM2 and V (Fig. 4A, B). Glomerulus DM2, but not $\mathrm{V}$, receives input from OSNs expressing the EB-sensitive receptor Or22a (Hallem and Carlson, 2006). Exposure to $10^{-1}$ EB was previously shown to induce enlargement of DM2, but not V, glomerulus (Sachse et al., 2007). Thus, we used glomerulus V here as an internal control. Volumes were measured in flies expressing ubiquitously the $n$-synaptobrevin-GFP reporter in the ENG3 transgenic line (Estes et al., 2000). In these animals, all synaptic neuropils were visible, hence all AL glomeruli. This allowed to estimate the relative AL volumes occupied by DM2 and V synaptic domains, and to normalize for possible AL size differences across genotypes or individuals. We first checked that expression of ENG3 in the GH298-Gal4 or krassavietz-Gal4 did not affect central adaptation (data not shown).

Coincident with normal adaptation, GH298/ENG3 flies exhibited a significant increase in the relative size of DM2 $(+17 \%$ on average), compared with solvent-exposed flies. As an internal control, the relative size of $\mathrm{V}$ did not change (Fig. 4C). By contrast, the odorant-specific glomerular size change induced by exposure was not detected when $P I 3 K$ was driven in GH298 (Fig. $4 D$ ) or $P I 3 K^{D N}$ in krasavietz (Fig. $4 E$ ). Since both manipulations are likely to alter the functional properties of the two types of neurons (see Discussion), we assessed glomerular size changes when both sets of LNs were manipulated simultaneously, i.e., expressing $P I 3 K^{D N}$ under the control of both drivers. Under this condition, central adaptation was restored and, likewise, the size of DM2 enlarged significantly $(+24 \%)$ while that of $\mathrm{V}$ remained unaffected (Fig. $4 F$ ), providing the morphological hall-mark for normal central adaptation.

Together, the data indicate that olfactory central adaptation requires a balance of excitation/inhibition activity by the local interneurons of the antennal lobe, and that it includes morphological changes in odorant-specific glomeruli.

\section{Discussion}

The dynamic properties of nervous systems are reflected on a variety of behavioral changes, including habituation and sensory adaptation, which are considered as "simple" (nonassociative) learning. Here, we study a process, phenomenologically distinct from these, that we called central adaptation (Devaud et al., 2001) 

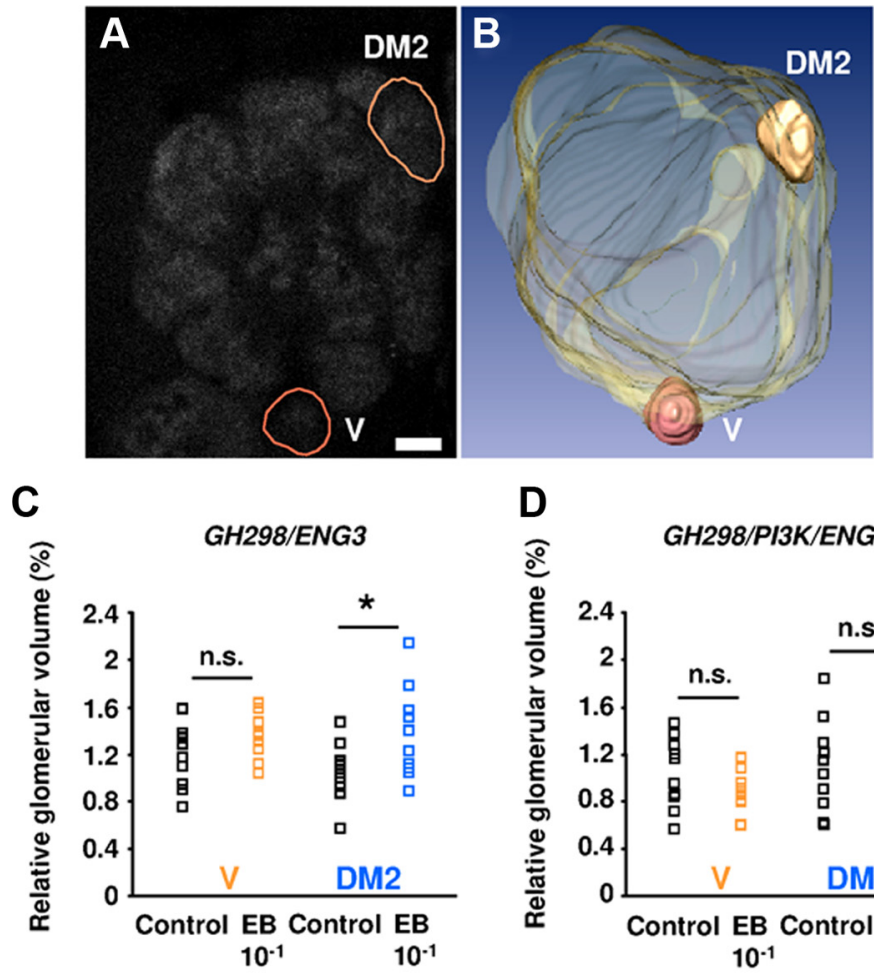

E

krasavietz/PI3K DN/ENG3

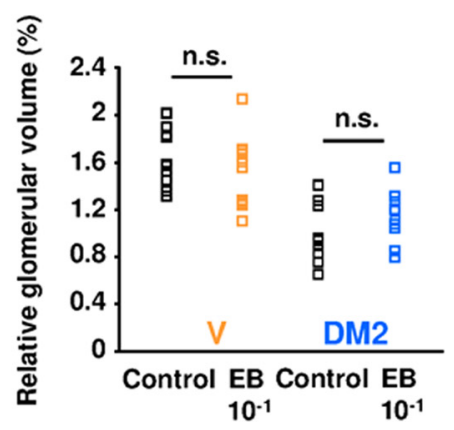

D

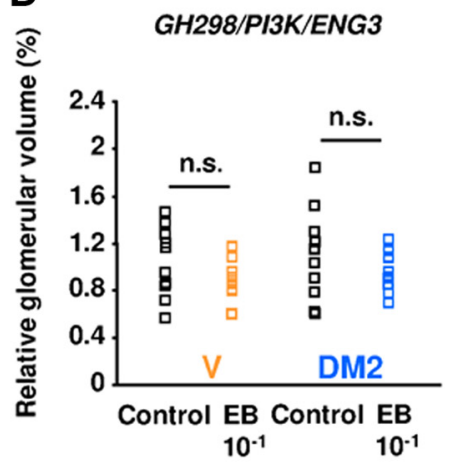

$\mathbf{F}$

krasavietz/GH298/PI3K DN/ENG3

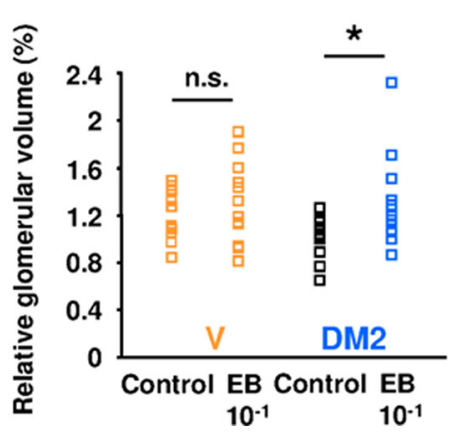

Figure 4. Structural effects of olfactory central adaptation. A, Frontal view (confocal section) of the right AL of a 6-d-old ENG3 fly. Glomeruli were detected by the $n$-synaptobrevin-GFP reporter. The two glomeruli (V and DM2) selected for volumetric measurements are outlined (brown lines). Scale bar, $10 \mu \mathrm{m} . B, 3 \mathrm{D}$ reconstruction of the selected glomeruli and their localization in the AL. C-F, Flies of the following genotypes: GH298-Gal4/ENG3 (C), GH298-Gal4/UASPI3K/ENG3 (D), krasavietz-Gal4/UASPI3KNN/ ENG3 (E), and krasavietz-Gal4/GH298-Gal4/UASPI3K $K^{D N} / E N G 3(\boldsymbol{F})$ were exposed to paraffin oil (control) or $10^{-1} \mathrm{~EB}$ (experimental) during days $2-5$, and their brains processed after the behavioral assay (day 6 ). Dot plots show volume measurements from $V$ (black and orange) and DM2 (black and blue) glomeruli. C, In exposed GH298-Gal4/ENG3 flies, DM2 showed a significant increment in volume, whereas V remained unaffected. No significant changes occurred in V or DM2 glomeruli when $G H 298$ (D) or krasavietz (E) neurons expressed the PI3K and PI3KDN constructs, respectively. Note that both genotypes were also ineffective to produce central adaptation. $\boldsymbol{F}$, In the double Gal4 driver genotype, however, the relative glomerular volume of DM2, but not that of $\mathrm{V}$, was increased in preexposed flies compared with controls (C). $n=10-12$ glomeruli/genotype/experimental group. n.s., Not significant.

and whose underlying cellular and molecular mechanisms were largely unknown. This form of experience-dependent plasticity appears as an adaptation of maturing brain networks to sensory input (Devaud et al., 2001; Sachse et al., 2007). Hence, it shares some features with classical forms of developmental plasticity such as those described originally by Hubel and Wiesel in the mammalian visual system, and whose neural bases are still under investigation (Smith and Bear, 2010).

Our results here indicate that olfactory central adaptation is a phenomenon developed primarily at the level of the ALs, rather than in the MBs, which are important brain centers for associa- tive forms of olfactory learning. Within antennal lobes, LNs, rather than PNs, seem to support the crucial events leading to central adaptation. The data also show that the phenomenon is prevented if the number of synapses is putatively changed, in either direction, in anyone of krasavietz and GH298 domains. In all cases, central adaptation is lost to the two odorants tested. Remarkably, normal central adaptation is restored when the genetic modification is elicited in the two LN subsets simultaneously. Furthermore, the morphological changes accompanying the phenomenon are consistent with the behavioral data. Since the krasavietz neurons are mostly excitatory and the GH298 mostly inhibitory, we hypothesize that the proper activation of PNs by these LNs requires a balance between excitatory and inhibitory inputs for central adaptation to take place. Electrophysiological recordings from PNs in animals where the LNs have been genetically modified would provide a direct answer to the question of what type of activity changes occur in the output PNs during this form of adaptation. The behavioral effects invite to predict that PN activity will differ if only one type of LNs had modified their numbers of synapses. Also, in the absence of genetic modifications, recordings from PNs should differ if only one type of LNs is directly stimulated. These expected results appear particularly relevant to be investigated in view of the strong electric coupling reported between eLNs and PNs but not so strong between iLNs and PNs (Yaksi and Wilson, 2010).

Although ultrastructural studies are still pending to provide the unequivocal demonstration for the connectivity among AL neurons, several electrophysiological studies offer the best available evidence (Huang et al., 2010; Seki et al., 2010; Yaksi and Wilson, 2010). We do not know whether the PI3K constructs used in this study affect the number and/or activity of electric contacts. Also, although eLNs in general show a strong chemical synaptic input onto iLNs, rather than on PNs, the firing properties of eLNs are celland odorant-specific. Previous studies (Yaksi and Wilson, 2010) have suggested that a major role of these neurons would be to recruit inhibition to the PNs, particularly when high odor intensities might lead to a saturation of PN activity. In turn, iLNs might maintain olfactory discrimination, consistently with their morphological and functional diversity (Chou et al., 2010; Seki et al., 2010). The lack of cross-adaptation indicates that olfactory discrimination is rather normal. Thus, keeping the balance between activities of both LN populations might provide a means to set gain control during exposure while preserving olfactory discrimination. 
Concerning the site in the $\mathrm{AL}$ where central adaptation is built, and notwithstanding the fact that the actual connectivity among AL neurons remains to be determined at the ultrastructural level, it is reasonable to assume that eLNs and iLNs converge, directly or indirectly, on PNs where the excitation/ inhibition ratio would be actually read. Alternatively, since eLNs and iLNs maintain reciprocal functional contacts, central adaptation could be built also through a direct interaction between eLNs and iLNs, perhaps through specific subsets of synapses, and the outcome would be later transferred to PNs. In either case, it seems clear that central adaptation requires an interaction between two opposing signals, excitation and inhibition, which should modify a molecular read-out mechanism located in the cell where both signals associate. As a result, avoidance toward high odorant concentrations is reduced. Our conclusions are consistent with the view that such avoidance would be primarily triggered by events in the AL (e.g., saturation of $\mathrm{PN}$ responses) rather than elsewhere (Asahina et al., 2009). In particular, our data do not show a crucial role for MB neurons in adaptation of the response following exposure, though further experiments based on complementary GAL4 lines expressed in different subsets of $\mathrm{MB}$ neurons would be necessary to confirm this. The requirement for a balanced connectivity between eLNs and iLNs may be important to adjust the system's response to modified sensory input: indeed, odor exposure during early adulthood can alter adult OSN responses (Iyengar et al., 2010). Here, we have not addressed the possible role of sensory input since we have targeted central neurons, but we do not exclude the possibility that peripheral receptor plasticity may contribute indirectly to the modifications of connectivity in central neurons shown here.

Interestingly, this eLN/iLN input ratio appears also relevant, in the same LNs, for olfactory sensory responses (Acebes et al., 2011). It should be noted that unexposed animals where such balance was disturbed still exhibit clear avoidance responses (Fig. 2). Thus, the lack of central adaptation in exposed animals is not a mere consequence of impaired olfactory perception. Rather, it seems that the balance is required for both the expression of olfactory responses and their adjustment to exposure. Whether this is a general requirement for other sensory modalities is still unknown. Even in the case of ocular dominance shift, a model of experience-dependent plasticity, the respective contributions of excitation and inhibition are still unsolved (for review, see Smith and Bear, 2010). In mammals, early monocular deprivation triggers synaptic rearrangements in the visual cortex, thus leading to a shift in ocular dominance (Wiesel and Hubel, 1963). While a weakening of excitatory input from the deprived eye has been considered for long as a major cause for decreased cortical responses to that eye, it is now clear that both excitatory and inhibitory neurons are affected. Moreover, a recent study has provided arguments in favor of a role of inhibitory connections (YazakiSugiyama et al., 2009). However, the importance of inhibition is still debated (Smith and Bear, 2010), and whether a balance with excitation is maintained still awaits experimental demonstration. Thus, it would be appropriate to investigate whether this is a general requirement for neural systems involved in perception or processing of other sensory modalities, in particular if it is a common property to the various forms of learning-related phenomena.

\section{References}

Acebes A, Ferrús A (2001) Increasing the number of synapses modifies olfactory perception in Drosophila. J Neurosci 21:6264-6273.

Acebes A, Martín-Peña A, Chevalier V, Ferrús A (2011) Synapse loss in olfactory local interneurons modifies perception. J Neurosci 31:2734-2745.
Asahina K, Louis M, Piccinotti S, Vosshall LB (2009) A circuit supporting concentration-invariant odor perception in Drosophila. J Biol 8:9.

Aso Y, Grübel K, Busch S, Friedrich AB, Siwanowicz I, Tanimoto H (2009) The mushroom body of adult Drosophila characterized by GAL4 drivers. J Neurogenet 23:156-172.

Berdnik D, Chihara T, Couto A, Luo L (2006) Wiring stability of the adult Drosophila olfactory circuit after lesion. J Neurosci 26:3367-3376.

Busto GU, Cervantes-Sandoval I, Davis RL (2010) Olfactory learning in Drosophila. Physiology (Bethesda) 25:338-346.

Chou YH, Spletter ML, Yaksi E, Leong JC, Wilson RI, Luo L (2010) Diversity and wiring variability of olfactory local interneurons in the Drosophila antennal lobe. Nat Neurosci 13:439-449.

Cuesto G, Enriquez-Barreto L, Caramés C, Cantarero M, Gasull X, Sandi C, Ferrús A, Acebes Á, Morales M (2011) Phosphoinositide-3-kinase activation controls synaptogenesis and spinogenesis in hippocampal neurons. J Neurosci 31:2721-2733.

Devaud JM, Acebes A, Ferrús A (2001) Odor exposure causes central adaptation and morphological changes in selected olfactory glomeruli in Drosophila. J Neurosci 21:6274-6282.

Estes PS, Ho GL, Narayanan R, Ramaswami M (2000) Synaptic localization and restricted diffusion of a Drosophila neuronal synaptobrevin-green fluorescent protein chimera in vivo. J Neurogenet 13:233-255.

Hallem EA, Carlson JR (2006) Coding of odors by a receptor repertoire. Cell 125:143-160.

Huang J, Zhang W, Qiao W, Hu A, Wang Z (2010) Functional connectivity and selective odor responses of excitatory local interneurons in Drosophila antennal lobe. Neuron 67:1021-1033.

Iyengar A, Chakraborty TS, Goswami SP, Wu CF, Siddiqi O (2010) Posteclosion odor experience modifies olfactory receptor neuron coding in Drosophila. Proc Natl Acad Sci U S A 107:9855-9860.

Laissue PP, Reiter C, Hiesinger PR, Halter S, Fischbach KF, Stocker RF (1999) Three-dimensional reconstruction of the antennal lobe in Drosophila melanogaster. J Comp Neurol 405:543-552.

Martín-Peña A, Acebes A, Rodríguez JR, Sorribes A, de Polavieja GG, Fernández-Fúnez P, Ferrús A (2006) Age-independent synaptogenesis by phosphoinositide 3 kinase. J Neurosci 26:10199-10208.

Olsen SR, Bhandawat V, Wilson RI (2007) Excitatory interactions between olfactory processing channels in the Drosophila antennal lobe. Neuron 54:89-103.

Olsen SR, Bhandawat V, Wilson RI (2010) Divisive normalization in olfactory population codes. Neuron 66:287-299.

Root CM, Semmelhack JL, Wong AM, Flores J, Wang JW (2007) Propagation of olfactory information in Drosophila. Proc Natl Acad Sci U S A 104:11826-11831.

Sachse S, Rueckert E, Keller A, Okada R, Tanaka NK, Ito K, Vosshall LB (2007) Activity-dependent plasticity in an olfactory circuit. Neuron 56:838-850.

Schwaerzel M, Heisenberg M, Zars T (2002) Extinction antagonizes olfactory memory at the subcellular level. Neuron 35:951-960.

Seki Y, Rybak J, Wicher D, Sachse S, Hansson BS (2010) Physiological and morphological characterization of local interneurons in the Drosophila antennal lobe. J Neurophysiol 104:1007-1019.

Shang Y, Claridge-Chang A, Sjulson L, Pypaert M, Miesenböck G (2007) Excitatory local circuits and their implications for olfactory processing in the fly antennal lobe. Cell 128:601-612.

Smith GB, Bear MF (2010) Bidirectional ocular dominance plasticity of inhibitory networks: recent advances and unresolved questions. Front Cell Neurosci 4:21.

Stocker RF, Heimbeck G, Gendre N, de Belle JS (1997) Neuroblast ablation in Drosophila P[GAL4] lines reveals origins of olfactory interneurons. J Neurobiol 32:443-456.

Tanaka NK, Ito K, Stopfer M (2009) Odor-evoked neural oscillations in Drosophila are mediated by widely branching interneurons. J Neurosci 29:8595-8603.

Wiesel TN, Hubel DH (1963) Single-Cell Responses in Striate Cortex of Kittens Deprived of Vision in One Eye. J Neurophysiol 26:1003-1017.

Wilson RI, Turner GC, Laurent G (2004) Transformation of olfactory representations in the Drosophila antennal lobe. Science 303:366-370.

Yaksi E, Wilson RI (2010) Electrical coupling between olfactory glomeruli. Neuron 67:1034-1047.

Yazaki-Sugiyama Y, Kang S, Câteau H, Fukai T, Hensch TK (2009) Bidirectional plasticity in fast-spiking GABA circuits by visual experience. Nature 462:218-221. 